\title{
VEHICLE ROUTING PROBLEM WITH SOFT TIME WINDOWS AND PATH CHOICE
}

\author{
Ali Gul Qureshi**, Sideney Schreiner**, Eiichi Taniguchi*** and Tadashi Yamada****
}

\section{Introduction}

The aim of city logistics is to fully optimize the urban freight movement with respect to the public and private costs and benefits including the fuel consumption and environmental costs ${ }^{1)}$. Most of the urban freight movement is primarily based on the truck traffic, which contributes to many urban traffic-related problems such as traffic congestion, parking issues, accidents and environmental problems. To counter these issues, city logistics suggests many measures and policies to reduce the number of trucks, their mileage and their idling times. The Vehicle Routing and scheduling Problem with Time Windows (VRPTW) is a typical route optimization technique employed in city logistics. It is also used to evaluate other city logistics measures relating to infrastructure planning and development (for example, optimal location of logistics terminals $\left.{ }^{2}\right)$ and infrastructure management (such as, cooperative delivery systems ${ }^{3)}$ ).

Most of the city logistics-related research utilizes the soft time windows variant of the VRPTW (abbreviated as VRPSTW), whereby an early arrival is penalized equal to the opportunity cost lost due to waiting and a high late arrival penalty is imposed on delayed service (delivery/pickup of goods). However, most of the exact optimization research has been directed towards the Vehicle Routing and scheduling Problem with Hard Time Windows (VRPHTW), though it lacks the practicality found in real life problems because of stringent restriction on even slight delays. Furthermore, these exact techniques allow waiting at no penalty cost in VRPHTW, which results in more waiting time as compared to the cases when waiting is penalized $^{4)}$ or even when waiting is allowed at no cost along with penalized late arrivals ${ }^{5}$. On the other hand, mostly heuristics (approximate) solutions have been used for the VRPSTW in city logistics-related research (for example see, Yamda et al., ${ }^{2)}$, Duin et al., ${ }^{6}$ ).

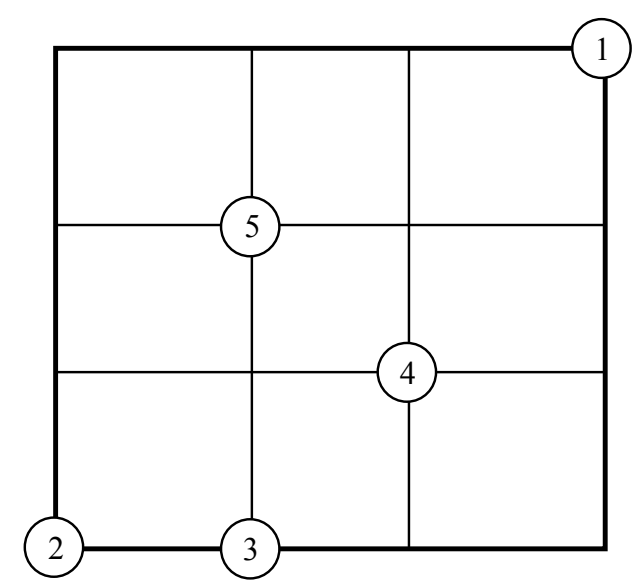

(a) Urban road network

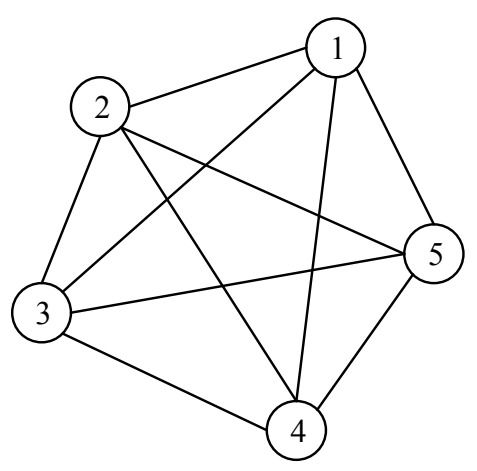

(b) A VRPTW instance

Figure 1 An urban road network and its transformation in a VRPTW instance

Moreover, in a real-life logistics instance, depot and pickup/delivery points (customers) may not be directly connected with each other using a single link of the urban road network. Rather their interconnectivity may contain various links possessing a variety of network attributes ${ }^{7)}$ and other dissimilarities such as, class of the road (highway or street, etc.), geometric aspects (width and number of lanes, etc.), tolled or un-tolled roads and municipal regulations (truck bans, etc.). Although, real urban road networks are

* Key words: logistics planning, logistics, path choice, soft time windows

** Student Member of JSCE, Dr. Eng., Post Doctoral Researcher, Faculty of Engineering, Kyoto University.

C-1 Kyotodaigaku Katsura, Nishikyo, Kyoto 615-8540, Tel. 075-383-3231, Fax. 075-950-3800.

*** Fellow Member of JSCE, Dr. Eng., Faculty of Engineering, Kyoto University.

C-1 Kyotodaigaku Katsura, Nishikyo, Kyoto 615-8540, Tel. 075-383-3229, Fax. 075-950-3800.

**** Full Member of JSCE, Dr. Eng., Faculty of Engineering, Kyoto University.

C-1 Kyotodaigaku Katsura, Nishikyo, Kyoto 615-8540, Tel. 075-383-3230, Fax. 075-950-3800. 
much complex, a typical example is given in Figure 1(a) for a grid shaped urban road network. Other node numbers except the depot (node 1) and customers (nodes 2 to 5), are not shown for the sake of clarity. On the other hand, the Vehicle Routing and scheduling Problem with Time Windows (VRPTW) is defined on a complete graph (i.e., a fully connected network), where each arc replaces a combination of actual urban road network links as shown in Figure 1(b). To fill this gap between practical and theoretic instances, a preprocessing stage of path choice between depot and customers and customer to customer is introduced. The path choice stage also faces some dilemmas such as, should it only be based on the myopic strategy of the shortest paths with respect to distance or travel time? or a network knowledge shall also be incorporated. The former is a network independent path, easy to calculate with standard shortest path algorithms, however, it is usually based on only one parameter of the network, for example links' length or travel time. On the other hand, the shortest path models, which incorporate network related issues and drivers' preferences (as used in this study) are typically based on local conditions and thus the parameters used in them needs to be reevaluated for the specific network under consideration.

It has been noticed that truck drivers often deviates from the most economical path between a pair of origin and destination ${ }^{8)}$. This is due to the drivers' choice, familiarity with the area or road and their own perception of cost of a certain link in the road network. For example, Figure 2 shows a computed cheapest cost (shown in red color and obtained using standard shortest path model based on generalized cost, for more details see $\S 4$ ); whereas, Figure 3 shows the observed path (shown in green color) followed by the truck driver taken from the dataset explained in $\S 4$. In this example, It can be noted that the observed path considerably deviates form the computed cheapest path, therefore, a vehicle route planning based on the latter would lead to erroneous estimates of costs.

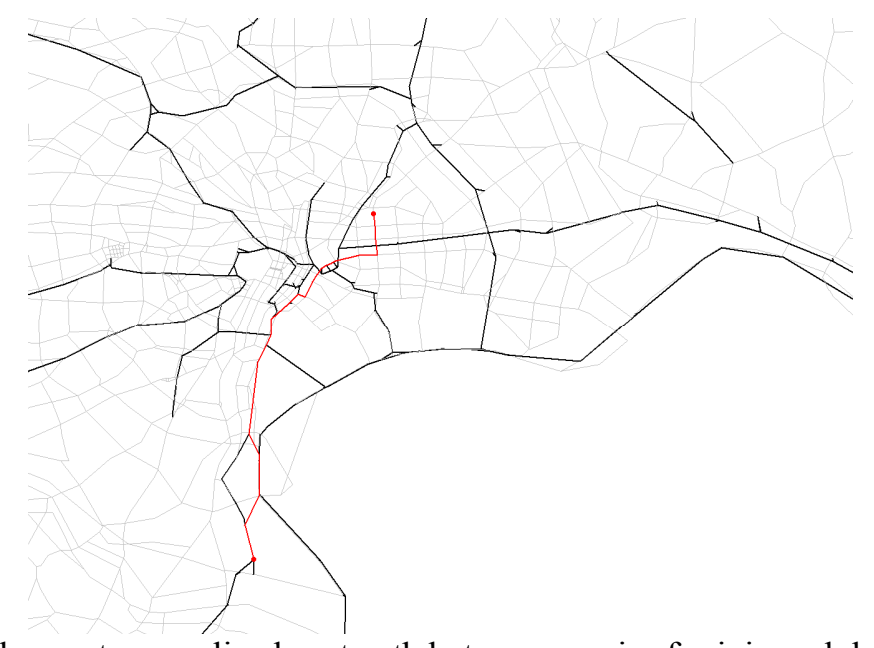

Figure 2 Cheapest generalized cost path between a pair of origin and destination

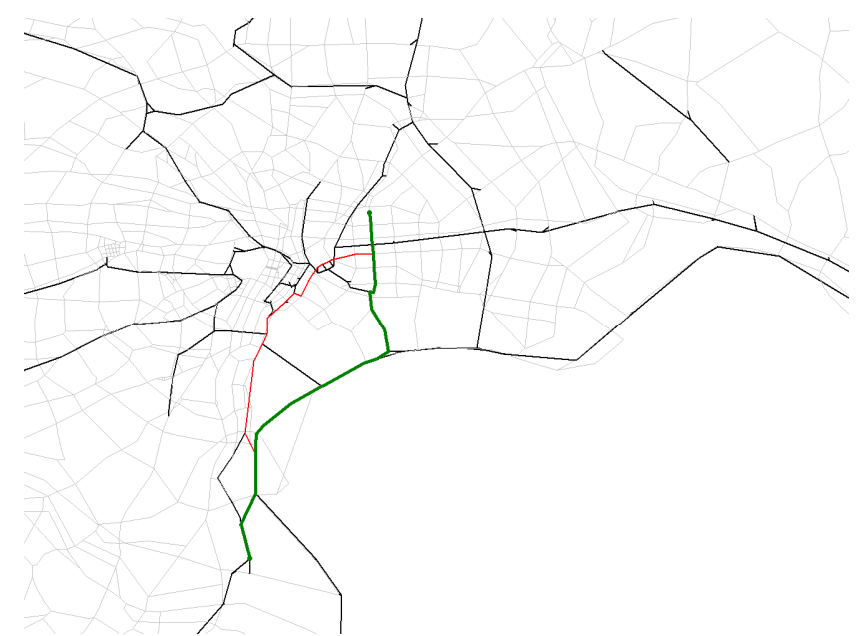

Figure 3 Observed path vs. the cheapest generalized cost path between a pair of origin and destination 
This paper presents an integration of an efficient path choice model presented by Schreiner et al., ${ }^{8)}$ that considers the drivers' perception and effects of various network related attributes (such as tolled/un-tolled road, restrictions for trucks), and an exact solution approach for the VRPSTW based on the Dantzig-Wolfe decomposition. The computational results show that the case that considers a simple recourse to implement a VRPSTW solution based on computed cheapest generalized cost without incorporating the effects of network attributes, results in significant cost overrun as compared to the route planning based on our proposed integrated approach. Another aspect of erroneous estimate of the cost along a path may be the uncertainty related to the estimate of travel time on the links, this situation is usually handled by the Dynamic Vehicle Routing and Scheduling with Time Windows (D-VRPSTW) or the Stochastic Vehicle Routing and Scheduling with Time Windows (S-VRPSTW), however, both of these models are not the subject area of this paper.

\section{Literature Review}

Usually, the path choice stage is based on the shortest path algorithms considering either the travel distance or travel times such as Dijkstra's algorithm ${ }^{9,10)}$. Ando et al., ${ }^{11)}$ used an ant colony system to obtain paths for construction of the VRPSTW instance's graph. The most related work to our approach, is the GIS-based decision support system "Map-Route" due to Ioannou et al. ${ }^{12)}$ that considered the Euclidean distances at the first stage and solved the VRPTW to form the complete graph (similar to the one shown in Figure 1(b)). The VRPTW routes are then mapped-back onto the real urban road network using shortest paths between the customers. The solution is then modified using knowledge based rules, which favour the main city arteries and roads with low traffic volumes as compared to regular/narrow roads and roads with high traffic volumes. Our path choice model considers the effects of various road network attributes in form of corresponding parameters to form the complete graph for the VRPSTW at the pre-processing stage. The model represents the drivers' perception of travel cost and shortest path based on a variety of network related attributes.

Many researchers have used heuristic techniques for the soft time windows environment with the idea to reduce the number of vehicles or overall solution $\operatorname{cost}^{13)}$. However, this study considers an exact solution approach for the VRPSTW based on the Dantzig-Wolfe decomposition (also called column generation), which has been successfully used for the exact solutions of the VRPHTW, in past. It decomposes the VRPTW in a set partitioning master problem and an Elementary Shortest Path Problem with Resource Constraints (ESPPRC) as its subproblem ${ }^{14}$. While the master problem remains the same, many researchers have worked with various shortest path variations as subproblems in their column generation schemes for the VRPHTW. For instance, Desrochers et al. ${ }^{15)}$ presented the first column generation based approach for the VRPHTW, using 2-cycle elimination while solving the relaxed shortest path subproblem. Irnich and Villeneuve ${ }^{16)}$ used a relaxed shortest path subproblem with $k$-cycle elimination (with $k \geq 3$ ) in their column generation scheme for the VRPHTW. The exact solution method used to solve the VRPSTW in this paper, also relies on the set partitioning formulation of the VRPTW but a different subproblem is solved as described in the next section.

Unlike our column generation scheme that iteratively adds the feasible routes of marginal negative cost from the subproblem to the set partitioning master problem, Calvete et al. ${ }^{17)}$ exploited goal programming to enumerate all the feasible routes in the first stage and then used the set partitioning problem to solve the VRP with soft time windows with a heterogeneous fleet and multiple objectives. In a similar approach, Fagerholt ${ }^{18)}$ solved a ship-scheduling problem with soft time windows. The Traveling Salesman Problem with Capacity, Hard Time Windows and Precedence Constraint (TSP-CHTWPC) was used to enumerate all feasible routes and then their schedules were optimized using soft time windows, before using a set partitioning problem. Therefore our approach gives an exact solution approach for the VRPSTW; whereas, the above cited references can be classified as heuristics.

\section{VRPSTW Model Formulation}

The VRPSTW is defined on a directed graph $G=(V, A)$. The vertex set $V$ includes the depot vertex 0 and the set of customers $C=\{1,2, \ldots, n\}$. The set $K$ represents the set of identical vehicles with capacity $q$ stationed at the depot. The arc set $A$ consists of all feasible arcs $(i, j), i, j \in V$. A cost $c_{i j}$ and a time $t_{i j}$ is associated with each arc $(i, j) \in A$. The time $t_{i j}$ includes the travel time on arc $(i, j)$ and service time at vertex $i$, while a fixed vehicle utilization cost is added to all outgoing arcs from the depot. With every vertex of $V$ associates a demand $d_{i}$ where $d_{0}=0$, and a time window $\left[a_{i}, b_{i}\right]$, which represents the earliest and the latest 
desired service start times. This study incorporates the soft time windows constraint by extending the latest service start time $b_{i}$ to $b_{i}{ }^{\prime}$ as shown in Figure 4, such soft time windows have been considered in the literature for the heuristic solution approaches (for example see Taillard et al. ${ }^{19)}$ and Gendreau et al. ${ }^{20)}$ ). The maximum penalty is considered equivalent to the cost of a dedicated single vehicle route only serving the concerned customer because it would be more cost effective to serve a customer individually if the amount of the late arrival penalty exceeds the cost of the single customer route. Taking $c_{l}$ as the unit late arrival penalty cost, $b_{i}{ }^{\prime}$ can be defined as eq. (1); where the first term ensures that if the vehicle visits the customer at $b_{i}^{\prime}$, it can return back to depot before its closing time and the second term adds as much as later arrival time to $b_{i}$ as allowed by the maximum late arrival penalty, described earlier. The arrival time-dependent cost is formulated as eq. (2), where $s_{j k}$ represents the arrival time of vehicle $k$ at customer $j$. In practice, it is very difficult to determine the exact value of the $c_{l}$, as penalty is usually not paid in terms of monetary units; it is often based on some interview data showing the preference of freight carriers.

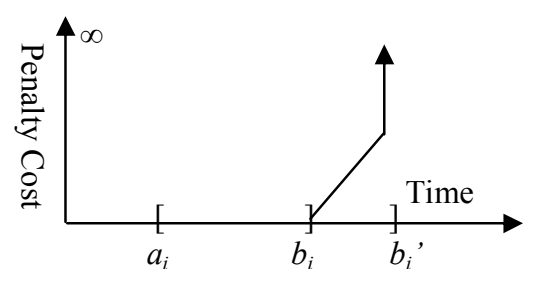

Figure 4 Penalty cost function for the VRPSTW

$$
\begin{aligned}
& b_{i}^{\prime}=\min \left[b_{0}-t_{i 0}, b_{i}+\frac{\left(c_{0 i}+c_{i 0}\right)}{c_{l}}\right] \\
& c_{i j k}^{\prime}=\left\{\begin{array}{l}
c_{i j}, \text { if } s_{j k} \leq b_{j} \\
c_{i j}+c_{l}\left(s_{j k}-b_{j}\right), \text { if } s_{j k}>b_{j}
\end{array}\right.
\end{aligned}
$$

Using the Dantzig-Wolfe decomposition the VRPSTW is formulated as

$$
\min \sum_{p \in P} c_{p} y_{p}
$$

subject to

$$
\begin{aligned}
& \sum_{p \in P} a_{i p} y_{p}=1, \quad \forall i \in C \\
& y_{p} \in\{0,1\} \quad \forall p \in P
\end{aligned}
$$

The master problem (eq.(3)- eq.(5)) consists of selecting a set of feasible paths of minimum cost generated by the Elementary Shortest Path Problem with Resource Constraints and Late Arrival Penalties (ESPPRCLAP) as the subproblem. The decision variable $y_{p}$ takes value 1 if the path $p \in P$ is selected and 0 otherwise, where $P$ is the set of all feasible paths. The number of times the path $p$ serves customer $i$ is represented by $a_{i p}$. A vector of dual variables (prices) is also generated in the master problem represented by $\pi_{i}$. Considering all identical vehicles and the reduced cost network, ESPPRCLAP can be formulated as:

$$
\begin{aligned}
& \min \sum_{(i, j) \in A} \overline{c_{i j}^{\prime}} x_{i j} \\
& \text { subject to } \\
& \sum_{i \in C} d_{i} \sum_{j \in V} x_{i j} \leq q \\
& \sum_{j \in V} x_{0 j}=1 \\
& \sum_{i \in V} x_{i h}-\sum_{j \in V} x_{h j}=0 \quad \forall h \in C
\end{aligned}
$$




$$
\begin{aligned}
& \sum_{i \in V} x_{i 0}=1 \\
& s_{i}+t_{i j}-s_{j} \leq\left(1-x_{i j}\right) M_{i j} \quad \forall(i, j) \in A \\
& a_{i} \leq s_{i} \leq b_{i}^{\prime} \quad \forall i \in V \\
& x_{i j} \in\{0,1\} \quad \forall(i, j) \in A
\end{aligned}
$$

The model contains two decision variables $x_{i j}$ which determines whether arc $(i, j)$ is used in the solution $\left(x_{i j}\right.$ $=1)$ or not $\left(x_{i j}=0\right)$ and $s_{j}$ that determines the service start time at vertex $j$ by the vehicle. Objective equation (6) minimizes the reduced cost of the path. $M_{i j}$ is a big constant. Constraint (7) is capacity constraint. Constraints (8)-(10) are flow conservation constraints. Constraint (11) is time windows constraint specifying that if a vehicle travels from $i$ to $j$, service at $j$ can not start earlier than that at $i$. Constraint (12) specifies the service start time at all vertices must be within their soft time windows $\left[a_{i}, b_{i}^{\prime}\right]$. Finally, the integrality of the decision variables is ensured using constraint (13).

The ESPPRCLAP subproblem is solved on the same network as VRPSTW, with the arcs cost being reduced by the corresponding dual variables (prices) (eq. (14)) generated in the master problem.

$$
\overline{c_{i j}^{\prime}}=c_{i j}^{\prime}-\pi_{i} \quad \forall i \in V
$$

\section{Path Choice Model}

The travel cost matrix $c_{i j}$ that appears in eq. (2) and again in its reduced version in the objective function of the ESPPRCLAP (eq. (6)), is based on the path choice between the depot and customers and customers to customers. As described in the literature review that mostly the Dijkstra's algorithm-based shortest paths are used at the path choice stage, which minimize the travel distance or the travel time. Instead of the shortest paths, the cheapest path concept can be considered based on the generalized cost (GC) that represents the cost to travel through one link as shown in eq. (15). The first term in eq. (15), considers the travel cost of the link $a$ based on the user's value of time (VOT), and the fuel consumption $\left(\right.$ Cons $\left._{a}\right)$, which is obtained using the average engine performance in liters per kilometer traveled, the fuel cost in monetary units per liter, and the link length in kilometers. As mentioned earlier that the drivers often deviates from the cheapest generalized cost paths (see, Figure 2 and Figure 3), therefore, this study adopts a path choice model presented by Schreiner et al., ${ }^{8}$ ) that considers the drivers' perception and effects of various network related attributes that modify the generalized cost (given by eq. (15)) of a link. The modified generalized cost is given by eq. (16).

$$
\begin{aligned}
& G C_{a}=\left(\text { Cons }_{a}+\text { VOT } \times \text { Time }_{a}\right) \\
& G C_{a}=\left(\text { Cons }_{a}+\text { VOT } \times \text { Time }_{a}\right) \times \prod_{k} \beta_{k}
\end{aligned}
$$

Where:

$\beta_{k}$ is the parameter for the $k$-th network attribute (e.g. road width)

Table 1 lists the network attributes considered in eq. (16), for example, links are distinguished whether they are tolled or un-tolled, located in CBD or within the ring road 7, number of lanes ( $\geq 4$ or less). Traffic regulations such as truck bans (based on their weight and height) have also been considered as significant parameters defining an actual truck route (as network attributes 5 and 6 in Table 1). The corresponding parameters $\left(\beta_{k}\right)$ were estimated for an extensive road network of Tokyo Metropolitan, consisting of 9231 nodes and 25062 links. Route duplication methodology is used for the calibration of route choice model (eq. (16)) using 597 observed truck paths between various origins and destinations (Figure 5) (for parameter estimation and further details, please see Schreiner et al., $\left.{ }^{8}\right)$. The VOT was estimated as 90 yen/minute for non-container trailer trucks; estimated values of other corresponding parameters for the network attributes, are also listed in Table 1. Figure 6 shows the path (shown in blue color) generated by the path choice model used in this study between the same origin and destination node as of Figures 2 and 3, it can be observed that it overlaps/matches the green path (observed/actual path) shown in Figure 3. The overall route duplication rate was about 0.8 for all the 597 observed truck paths for the estimated values of $\beta_{k}$ given in Table 1. It may 
be noted that the cost of the path shown in Figure 6, given by eq. (16) is not the actual cost of that path, it just represents the driver's preference given to the cost of each link on that path (shown in Figure 6). The actual cost of the path (shown in Figure 6) is then calculated separately using eq. (15) to be used in the VRPSTW model.

Table 1 Network Attributes Estimated for Road Network of Tokyo Metropolitan

Attribute Estimated values

\begin{tabular}{lll} 
& & of $\beta_{k}$ \\
\hline 1 & Tolled road & 0.6 \\
2 & CBD area & 1.0 \\
3 & Ring Road 7 & 0.9 \\
4 & Number of lanes & 0.6 \\
5 & Heavy truck permission & 0.8 \\
$\mathbf{6}$ & Tall truck permission & 0.7 \\
\hline
\end{tabular}

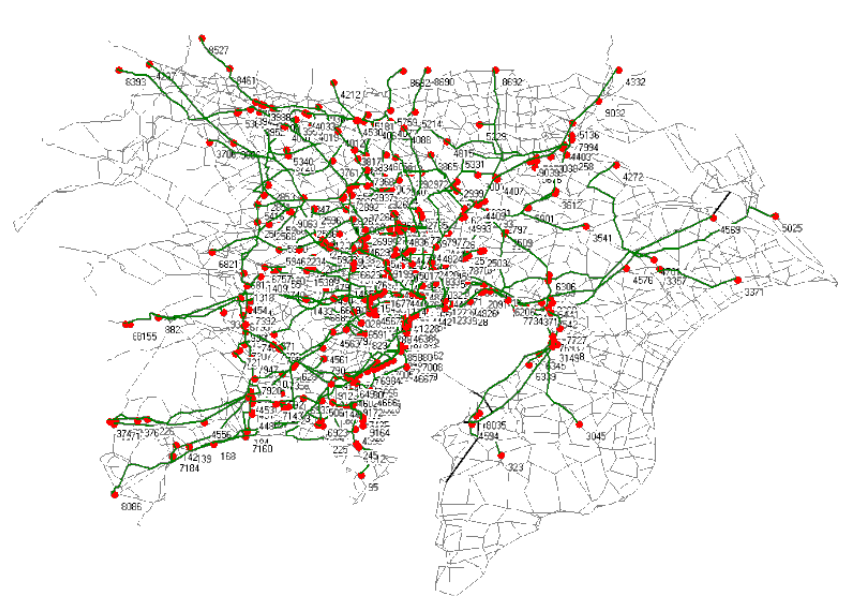

Figure 5 Observed path between various pairs of origin and destinations

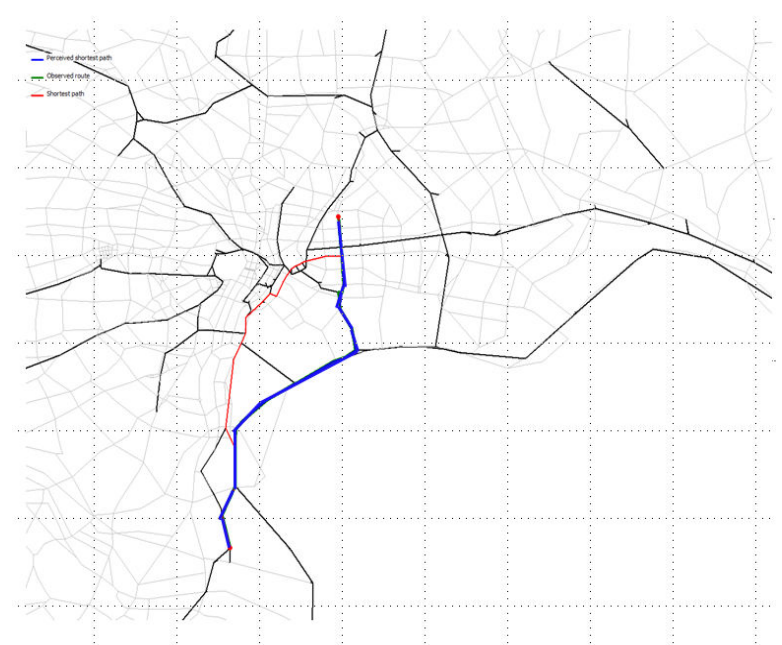

Figure 6 Path given by the used path choice model between same pairs of origin and destinations as of Figures 2 and 3

\section{Test Instances}

The hypothetical test instances are based on the Tokyo metropolitan road network (Figure 5). The test instances consists 25 customer nodes and a depot node. Two basic datasets were generated having different geographic locations of the customers and depots (Figure 7 and Figure 8), generated randomly. Customers 
are located geographically close to each other in customer dataset 1 (CS1) relative to those in customer dataset 2 (CS2). Within each customer datasets five different test instances were generated, each with a randomly generated different time windows pattern (i.e., in different test instance the same customer location was assigned different start and end times of time windows). The nomenclature of the test instances CSx-25$y$ depicts that the instance belongs to customer dataset $\mathrm{x}$ and its unique ID is $y$. The width of time windows (i.e., $b_{i}-a_{i}$ ) was kept as 10 minutes in all instances, so was the service time at each customer. Large computation time is required, if the time windows are fully relaxed (using eq. (1)) $)^{21)}$, therefore, time windows at customers were relaxed for 10 minutes only (i.e., $b_{i}{ }^{\prime}-b_{i}=10$ ).

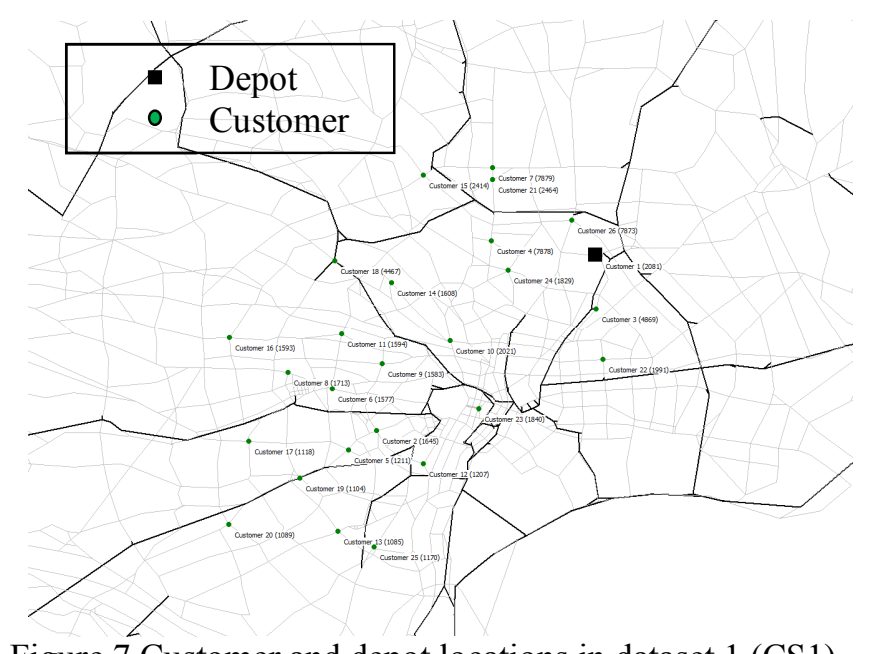

Figure 7 Customer and depot locations in dataset 1 (CS1)

Three different case scenarios were constructed to test the performance of our proposed integrated approach of the used path choice model and the VRPSTW exact solution against the usually adopted combination of Dijkstra's algorithm based path choice and VRPSTW exact solution.

Case I: The cost matrix $\left(c_{i j}\right)$ and the travel time matrix $\left(t_{i j}\right)$ of the VRPSTW model ( $\left.\$ 3\right)$ were based on Dijkstra's algorithm, minimizing the generalized cost given by eq. (15). This is the base scenario representing the usual case of using "calculated paths" (similar to the path in Figure 1) at the path choice stage in the vehicle route planning without considering the network attributes or the truck drivers' choice.

Case II: This scenario implements the vehicle route planning of Case I based on the cost matrix and travel time matrix of paths obtained using the path choice model adopted in this study. Realizing the fact that the path choice model used in this study tries to duplicate actual observed truck paths (similar to the path in Figure 2), this scenario mimics the situation similar to the real-life, where the drivers follow a given vehicle route plan (order of the customers) using the paths of their own choice, between each pair of customers. As these paths are sometime different than the calculated paths (used in Case I), the vehicle route planning of Case I may not be feasible to implement due to the violation of even relaxed time windows at a customer along a vehicle route (i.e. the vehicle arrives later than $b_{i}{ }^{\prime}$ at customer $i$ ). In such situation a simple recourse policy has been adopted; a new truck is dispatched from the depot to cover the remaining part of the route (i.e. from customer $i$ onwards) while the first truck returns to the depot after completing the service at the predecessor customer of customer $i$. Similar recourse policy is commonly adopted in stochastic vehicle routing problem, in case of route failures (for example see, Tan et al., ${ }^{22)}$; Gendreau et al. ${ }^{23)}$ ).

Case III: The cost matrix $\left(c_{i j}\right)$ and the travel time matrix $\left(t_{i j}\right)$ of the VRPSTW model ( $\left.\$ 3\right)$ were based on the path choice model adopted in this study, which minimizing the generalized cost given by eq. (16) in order to find paths similar to the observed truck paths. This scenario is the outcome of the proposed integration of the path choice model and the VRPSTW solution, which try to incorporate the driver's perception (in the sense that the $\beta_{k}$ values are based on the observed truck paths coming from the driver's own perception of generalized cost of a link, as given in Table 1) in the vehicle route planning. It should be noted that, only the paths (i.e. the road network's link sequence) of the path choice model that uses eq. (16) were adopted in Cases II and III, their cost and travel time were re-calculated using the un-factored generalized cost given by eq. (15) to get the actual cost and travel time along these paths. 


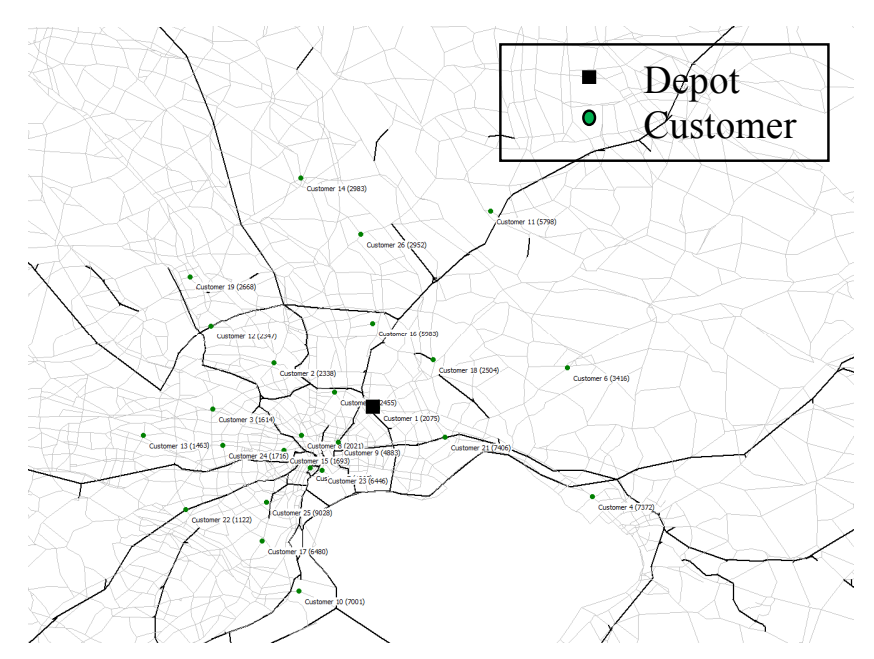

Figure 8 Customer and depot locations in dataset 2 (CS2)

\section{Results and Discussion}

Table 2 summarizes the results of various cases for the test instances. Columns 2 and 3 give the number of vehicle required and the cost in Japanese Yen (JPY) in Case I; columns 4 and 5 gives the corresponding results when the vehicle route planning of Case I is implemented in Case II as described above, and column 6 gives the consequent percentage increase in the cost. Columns 7 and 8 reports the number of vehicles and cost of the solution for the proposed integrated approach, and column 9 gives the percentage increase in cost as compared to the base case, i.e. the Case I. From the results of Case II, it can be observed that the vehicle route planning of Case I may loose its efficiency considerably, if the drivers choose their own path between the customers along a vehicle route (which often is the case in urban logistics). The average cost overrun was found to be $15.3 \%$ in customer dataset 1 (CS1) instances, moreover, this cost overrun is totally un-expected at the time of vehicle route planning, which considers that the drivers are fully aware of actual generalized cost of each link and their perception of the generalized cost is not effected by other factors. On the other hand, if the truck drivers' perception of the generalized cost and the resulting paths are incorporated within the vehicle route optimization (as in Case III), the cost overruns are fairly low, averaging at around $5.0 \%$ for the test instances belonging to the CS1. This low cost overrun is fully known at the time of planning and thus could be considered as the actual cost instead the cost of Case I. Similar results obtained for the test instances belonging to the CS2, show that the excessive cost overrun in Case II are not instance specific. Results of CS1-25-2 presents a possible scenario where the cost of both Case II and Case III is the same.

Table 2 Summary of the VRPSTW solutions in Case I, II and III

\begin{tabular}{|c|c|c|c|c|c|c|c|c|}
\hline \multirow{2}{*}{$\begin{array}{c}\text { Test } \\
\text { Instance } \\
\text { (1) }\end{array}$} & \multicolumn{2}{|c|}{ Case I } & \multicolumn{4}{|c|}{ Case II } & \multicolumn{2}{|c|}{ Case III } \\
\hline & $\begin{array}{l}\text { No. of } \\
\text { vehicles } \\
\text { (2) }\end{array}$ & $\begin{array}{c}\text { Cost } \\
\text { (JPY) } \\
\text { (3) }\end{array}$ & $\begin{array}{c}\text { No. of } \\
\text { vehicles } \\
\text { (4) }\end{array}$ & $\begin{array}{c}\text { Cost } \\
\text { (JPY) } \\
(5)\end{array}$ & $\begin{array}{c}\% \\
\text { increase } \\
(6)\end{array}$ & $\begin{array}{c}\text { No. of } \\
\text { vehicles } \\
\text { (7) }\end{array}$ & $\begin{array}{c}\text { Cost } \\
\text { (JPY) } \\
\text { (8) }\end{array}$ & $\begin{array}{c}\% \\
\text { increase } \\
(9)\end{array}$ \\
\hline CS1-25-1 & 6 & 224632.3 & 6 & 231028.3 & 2.8 & 6 & 230732.8 & 2.7 \\
\hline CS1-25-2 & 6 & 223927.5 & 6 & 227618.4 & 1.6 & 6 & 227618.4 & 1.6 \\
\hline CS1-25-3 & 6 & 224908.9 & 7 & 259001.9 & 15.2 & 6 & 232265.9 & 3.3 \\
\hline CS1-25-4 & 5 & 199924.6 & 7 & 258107.2 & 29.1 & 5 & 208726.1 & 4.4 \\
\hline \multirow[t]{2}{*}{ CS1-25-5 } & 5 & 201666.6 & 7 & 257585.4 & 27.7 & 6 & 227733.1 & 12.9 \\
\hline & & & \multicolumn{2}{|c|}{ Average } & 15.3 & \multicolumn{2}{|c|}{ Average } & 5.0 \\
\hline CS2-25-1 & 9 & 389225.1 & 11 & 470819.3 & 21.0 & 10 & 429940.9 & 10.5 \\
\hline CS2-25-2 & 13 & 502207.4 & 14 & 543792.3 & 8.3 & 13 & 504367.7 & 0.4 \\
\hline CS2-25-3 & 9 & 381728.9 & 11 & 451953.7 & 18.4 & 10 & 415430.5 & 8.8 \\
\hline CS2-25-4 & 9 & 404955.9 & 11 & 475222.1 & 17.4 & 10 & 437140 & 7.9 \\
\hline \multirow[t]{2}{*}{ CS2-25-5 } & 9 & 372594.6 & 10 & 423976.3 & 13.8 & 9 & 392755.4 & 5.4 \\
\hline & & & \multicolumn{2}{|c|}{ Average } & 15.8 & \multicolumn{2}{|c|}{ Average } & 6.6 \\
\hline
\end{tabular}


Table 3 Details of the VRPSTW solutions in Case I, II and III

\begin{tabular}{|c|c|c|c|c|c|c|c|c|}
\hline \multirow{2}{*}{$\begin{array}{c}\text { Test } \\
\text { instance }\end{array}$} & \multicolumn{2}{|c|}{ Case I } & \multicolumn{2}{|c|}{ Case II } & \multicolumn{2}{|c|}{ Case III } & \multirow[b]{2}{*}{$\begin{array}{l}\text { \% increase in } \\
\text { delivery time } \\
\text { (Case III vs. } \\
\text { Case II) } \\
(8)\end{array}$} & \multirow[b]{2}{*}{$\begin{array}{l}\% \text { increase in } \\
\text { late arrival } \\
\text { time (Case III } \\
\text { vs. Case II) } \\
(9)\end{array}$} \\
\hline & $\begin{array}{l}\text { Delivery } \\
\text { time } \\
\text { (2) } \\
\end{array}$ & $\begin{array}{l}\text { Late } \\
\text { arrival } \\
\text { time } \\
(3) \\
\end{array}$ & $\begin{array}{l}\text { Delivery } \\
\text { time } \\
(4) \\
\end{array}$ & $\begin{array}{l}\text { Late } \\
\text { arrival } \\
\text { time } \\
(5) \\
\end{array}$ & $\begin{array}{l}\text { Delivery } \\
\text { time } \\
(6)\end{array}$ & $\begin{array}{l}\text { Late } \\
\text { arrival } \\
\text { time } \\
(7) \\
\end{array}$ & & \\
\hline CS1-25-1 & 899.10 & 13.87 & 944.26 & 20.39 & 965.29 & 14.15 & 2.2 & -30.6 \\
\hline CS1-25-2 & 931.83 & 13.73 & 968.11 & 14.07 & 968.11 & 14.07 & 0.0 & 0.0 \\
\hline CS1-25-3 & 945.92 & 8.91 & 1021.74 & 13.24 & 991.11 & 11.86 & -3.0 & -10.4 \\
\hline CS1-25-4 & 916.64 & 18.54 & 1035.42 & 9.29 & 963.01 & 30.61 & -7.0 & 229.3 \\
\hline CS1-25-5 & 877.26 & 30.57 & 1033.07 & 12.40 & 1001.44 & 8.08 & -3.1 & -34.8 \\
\hline CS2-25-1 & 1617.93 & 23.69 & 1857.10 & 25.91 & 1743.00 & 12.06 & -6.1 & -53.5 \\
\hline CS2-25-2 & 1815.16 & 7.44 & 1867.83 & 1.30 & 1767.96 & 1.30 & -5.3 & 0.0 \\
\hline CS2-25-3 & 1515.18 & 28.67 & 1643.57 & 24.20 & 1574.81 & 18.18 & -4.2 & -24.9 \\
\hline CS2-25-4 & 1695.76 & 40.91 & 1885.43 & 31.34 & 1792.73 & 34.06 & -4.9 & 8.7 \\
\hline CS2-25-5 & 1507.59 & 11.40 & 1657.07 & 20.56 & 1601.12 & 23.86 & -3.4 & 16.1 \\
\hline
\end{tabular}

A change in the number of vehicles required in Case I and Case II, shows the functioning of the recourse policy, described in the previous section. Case II required two additional vehicles in five instances (out of ten) and one additional vehicle in three other instances; whereas, the Case III resulted in one additional vehicle only in three test instances (out of ten). Similar to the case of cost overrun, the additional vehicles required in Case III are fully known at the time of planning, whereas, in Case II, dispatch of additional vehicles has to be done dynamically. This situation would lead to better vehicle capacity management in Case III as compared to Case II, furthermore, it will also result in better optimization of the delivery time as shown by column (8) of Table 3 . The delivery time includes the time required by vehicles to travel along their designated routes plus the service time at the customers they serve.

Columns (2)-(7) in Table 3 give the details of solutions, i.e. the delivery time and the late arrival time for all the three cases, and columns (8) and (9) show the percentage increase in delivery time and late arrival time in Case III as compared to Case II, respectively. Case III resulted in slightly increased delivery time in only one test instance, whereas, the delivery time of Case II is considerably high in eight test instances. Although, the recourse policy followed in Case II dispatches a new vehicle from the depot when the late arrival time at a particular customers is very high resulting in vehicle arrival after $b_{i}{ }^{\prime}$, even this policy seems to be inefficient in reducing the overall late arrival time in Case II as compared to the Case III, which was able to produce solutions with comparatively less late arrival time in five test instances and matched the late arrival time of Case II in two other test instances. This, once again, proves the importance of considering the longer lengths of the paths to be followed by the drivers instead of the shortest (cheapest) paths at the planning stage (as in Case III), rather than at the implementation stage (as in Case II).

It can be noted that the average increase in cost in Case II and Case III as compared to the cost of Case I is slightly higher in dataset CS2 as compared to the CS1, this could be due to the fact that the customer locations in CS2 are dispersed having longer paths between them that might lead to more deviations (in Case II and Case III) from the computed shortest paths (Case I). On the other hand, if the customers are located far apart, there is an increased possibility that the cheapest path (Case I) consists of many tolled links, which are also favored in the paths generated by the used path choice model and hence this situation could lead to less cost differences among the three cases. It can also be observed that, even though the customer location is same in all the instances in a particular series (for example in CS1-25-x) various instances belonging to that series have very different results (costs and vehicle requirements), which is entirely attributed to the different time windows patterns making them completely different instances. Similarly, the extent of time windows relaxation (i.e., $b_{i}{ }^{\prime}-b_{i}$ ) can also effect the feasibility of a route and thus can affect the increase in cost in Case II and Case III as compared to Case I.

\section{Conclusion}

An integration of exact VRPSTW solution and an efficient path choice model is developed that considers the network attributes and truck drivers' perceptions of the generalized cost of a link, at the path choice stage 
of the vehicle route planning. Computational results showed that the vehicle route planning based on the path choice models which ignore such effects, may result in unexpected heavy cost overruns and may require dispatch of additional vehicles in dynamic time to restore the feasibility of routes. On the other hand the proposed planning based on the integrated approach would incorporate the unexpected cost overruns and additional vehicles (if any) in the planning process. It was found that the average cost overrun under such integrated planning approach is significantly less as compared to the planning approach based on commonly used pure shortest path algorithms without considering the network attributes or the truck drivers' perception.

\section{References:}

1) Taniguchi, E., Thompson, R.G., Yamada, T. and Van Duin J.H.R.: City Logistics; Network Modeling and Intelligent Transport Systems, Pergamon, Oxford, 2001.

2) Yamada, T., Taniguchi, E. and Itoh, Y.: Co-operative vehicle routing model with optimal location of logistics terminals, in Taniguchi, E., \& Thompson, R. G., eds., City Logistics II, pp. 139-153, Institute for City Logistics, Japan, 2001.

3) Qureshi, A., \& Hanaoka, S.: Analysis of the effects of cooperative delivery system in Bangkok, in Taniguchi, E., and Thompson, R.G., eds., Recent Advances in City Logistics Proceedings of the 4th International Conference on City Logistics, Langkawi, Malaysia, 2005, 59-73, Elsevier, Oxford, 2005.

4) Qureshi, A.G., Taniguchi, E. and Yamada, T.: Effects of relaxing time windows on vehicle routing and scheduling, Infrastructure Planning Review, Vol. 24, pp. 927-936, 2007.

5) Qureshi, A.G., Taniguchi, E. and Yamada, T.: A comparison of environmental impacts of hard time windows and semi soft time windows using exact routing solution, Infrastructure Planning Review, Vol. 25, pp. 451-462, 2008.

6) Duin, van.J.H.R., Tavasszy, L.A. and Taniguchi E.: Real time simulation of auctioning and re-scheduling process in hybrid freight markets, Transportation Research Part B, Vol. 41, pp. 1050-1066, 2007.

7) Hyodo, T., Suzuki, N. and Takahashi, K.: Modeling of bicycle route and destination choice behavior for bicycle road network plan, Transportation Research Record, No. 1705, pp. 70-76, 2000.

8) Schreiner, S., Hyodo, T. and Takahashi, Y.: A study on the influence of network attributes on the route choice behavior, Journal of the Eastern Asia Society for Transportation Studies, Vol. 7, pp. 642-653, 2007.

9) Qureshi, A.G., Taniguchi, E. and Yamada, T.: Elementary shortest path problem with resource constraints and time dependent late arrival penalties, JSCE Journal Division D, Vol.63, pp. 579-590, 2007.

10) Prindezis, N., Kiranoudis, C.T. and Marinos-Kouris, D.: A business-to-business fleet management service provider for central food market enterprises, Journal of Food Engineering, Vol. 60, pp. 203-210, 2003.

11) Ando, N., Taniguchi, E., Yamada, T. and Oka, G.: Evaluation on route travel time with meanvariance approach (in Japanese), Proceedings of the 28th JSTE Annual Conference, pp. 173-176, 2008.

12) Ioannoue, G., Kritikos M.N. and Prastacos, G.P.: A GIS-based decision support system for intra-city vehicle routing with time windows, The Journal of the Operational Research Society, Vol. 53, pp. 842854, 2002.

13) Taniguchi, E., \& Thompson, R.G.: Modeling city logistics, Transportation Research Records, Vol. 1790, pp. 45-51. 2002.

14) Feillet, D., Dejax, P., Gendreau, M. and Gueguen, C.:An exact algorithm for the elementary shortest path problem with resource constraints: Application to some vehicle routing problems, Networks, pp. 216229, 2004.

15) Desrochers, M., Desrosiers, J. and Solomon, M.: A new optimization algorithm for the vehicle routing problem with time windows, Operations Research, Vol. 40(2), pp. 342-354, 1992.

16) Irnich, S., \& Desaulniers, G.: Shortest path problems with resource constraints, in G. Desaulniers, Desrosiers, J., \& Solomon M.M., eds., Column Generation, Springer, New York, pp. 33-65, 2005.

17) Calvete, H.I., Gale, C., Oliveros M.J. and Valverde, B.S.: A goal programming approach to vehicle routing problem with soft time windows, European Journal of Operational Research, Vol. 177, pp. 17201733, 2007.

18) Fagerholt, K.: Ship scheduling with soft time windows: An optimisation based approach, European Journal of Operational Research, Vol. 131, pp. 559-571, 2001. 
19) Taillard, E., Badeau, P., Guertin, F., Gendreau, M. and Potvin, J.: A tabu search heuristic for the vehicle routing problem with soft time windows, Transportation Science, Vol. 31, pp. 170-186, 1997.

20) Gendreau, M., Guertin, F., Potvin, J. and Taillard, E.: Parallel tabu search for real-time vehicle and dispatching, Transportation Science, Vol. 33, pp. 381-390, 1999.

21) Qureshi, A.G., Taniguchi, E. and Yamada, T.: An exact solution approach for vehicle routing and scheduling problems with soft time windows, Transportation Research Part E, Vol. 45, pp. 960-977, 2009.

22) Tan, K.C., Cheong, C.Y. and Goh, C. K.: Solving multiobjective vehicle routing problem with stochastic demand via evolutionary computation, European Journal of Operational Research, Vol. 177, pp. 813839, 2007.

23) Gendreau, M., Laporte, G. and Seguin R.: An exact algorithm for the vehicle routing problem with stochastic demands and customers, Transportation Science, Vol. 29, pp. 143-155, 1995.

\section{VEHICLE ROUTING PROBLEM WITH SOFT TIME WINDOWS AND PATH CHOICE} Ali Gul Qureshi**, Sideney Schreiner**, Eiichi Taniguchi*** and Tadashi Yamada****

This paper presents an integration of an efficient path choice model that considers the drivers' perception and effects of various network related attributes (such as tolled/un-tolled road, restrictions for trucks), and an exact solution approach for the Vehicle Routing and scheduling with Soft Time Windows (VRPSTW) based on the Dantzig-Wolfe decomposition (commonly known as the column generation). It was found that the average cost overrun under such integrated planning approach is significantly less as compared to the planning approach based on commonly used pure shortest path algorithms without considering the network attributes or the truck drivers' perception. 